\title{
Characteristics of ventilator-associated pneumonia due to hypervirulent Klebsiella pneumoniae genotype in genetic background for the elderly in two tertiary hospitals in China
}

Chao Liu' ${ }^{1}$ and Jun Guo $2,3^{*}$

\begin{abstract}
Background: Aerobactin is a critical factor for the hypervirulent Klebsiella pneumoniae (hvKp), but data for the aerobactin-positive genotype of hvKp in elderly persons with ventilator-associated pneumonia (VAP) is limited. The purpose of this study is to understand the risk factors and characteristics of the hvKp genotype for elderly patients with VAP.

Methods: A retrospective study of 73 elderly patients with Kp was conducted from November 2008 to December 2017 in two tertiary hospitals. The clinical and microbiological data, including inflammatory reaction, nutritional status, antimicrobial susceptibility testing, string test, extended-spectrum- $\beta$-lactamase (ESBL) production, virulenceassociated gene (capsular serotype-specific gene and rmpA/A2,magA,aerobaction) and multilocus sequence typing, of the hvKp group defined as aerobactin positive were compared with those of classic Kp strains.

Results: Of 73 Kp isolates, 46.6\% were hvKp. ST23 is highly prevalent in two hospitals but is not highly associated with hvKp in different hospitals. Additionally, ST23, ST37 and ST2906 are more likely to induce lethal VAP. Most hvKp strains are sensitive to common antibiotics, but the number of multidrug-resistant (MDR) hvKp is increasing. Importantly, 38.2\% of hvKp isolates produced ESBLs. Hypermucoviscosity and virulence-associated genes (K1, magA and rmpA/A2) were highly clustered in the hvKp group $(P<0.001)$. Cancer $(P=0.004)$, digestive disease $(P=0.038)$ and surgery $(P=0.023)$ within 1 month are strongly associated with the VAP-hvKp group. The incidence of septic shock $(P=0.016)$ and Sequential Organ Failure Assessment (SOFA) scores $(P<0.001)$ are significantly higher in the hvKp group. Multivariate analysis indicated that cancer (odds ratio $[\mathrm{OR}]=5.365$ ) is an independent risk factor for VAP-hvKp infection.
\end{abstract}

Conclusions: The morbidity for elderly patients with VAP due to hvKp is high. MDR-HvKp is emerging, which is a great challenge for clinical practice.

Keywords: Klebsiella pneumoniae, Hypervirulent, Aerobactin, Risk factor, ESBL-hvKp, Ventilator-associated pneumonia

* Correspondence: guojunhx301@163.com

2Department of Respiratory Medicine, School of Clinical Medicine, Beijing

Tsinghua Changgung Hospital, Tsinghua University, Beijing, China

${ }^{3}$ Department of Geriatric Respiratory Medicine, Chinese PLA General Hospital,

Beijing, China

Full list of author information is available at the end of the article

(c) The Author(s). 2018 Open Access This article is distributed under the terms of the Creative Commons Attribution 4.0 International License (http://creativecommons.org/licenses/by/4.0/), which permits unrestricted use, distribution, and reproduction in any medium, provided you give appropriate credit to the original author(s) and the source, provide a link to the Creative Commons license, and indicate if changes were made. The Creative Commons Public Domain Dedication waiver (http://creativecommons.org/publicdomain/zero/1.0/) applies to the data made available in this article, unless otherwise stated. 


\section{Background}

Ventilator-associated pneumonia (VAP) is the most frequent life-threatening nosocomial infection in critically ill patients $[1,2]$. Klebsiella pneumoniae (Kp) is one of the most common gram-negative bacteria causing hospital infections, especially various notoriously fatal infections. Kp includes two distinct groups: hypervirulent (hvKp) and classical (cKp). One of the characteristics of cKp is acquiring an antibiotic-resistant gene. The other type is hvKp, which is traditionally defined as hypermucoviscosity by string test, inducing aggressive invasive community infection, bloodstream infection, and pyogenic liver abscesses (PLA) for immunocompetent ambulatory younger adults with no underlying diseases [36]. However, the definition of hvKp by string test is controversial [7, 8]. Previous studies showed that certain hypermucoviscous $K$. pneumonia (hmvKp) strains are not closely related to high virulence in in vitro and in vivo models $[7,8]$. Thus, to differentiate hvKp from cKp by the hypermucoviscosity phenotype alone may be inappropriate $[9,10]$. Recently, aerobactin, a dominant component of the siderophore system, has been established as a critical virulence factor for the hvKp genotype $[3,10,11]$. A previous multi-centre study in China targeting middle-aged patients, illustrated the clinical and molecular characteristics of hvKp (defined as aerobactin positive) infection [10]. However, the data for the hospital infection in the elderly with hvKp VAP, who commonly suffer from various underlying disease and nutritional complications, is rare.

Although most previous studies demonstrated that hvKp is sensitive to most antibiotics, which is prominently different from cKp strains, the multi-drug resistance (MDR) and extended-spectrum- $\beta$-lactamase (ESBL)-producing hvKp, especially those resistant to colistin and carbapenems, are emerging in China [12-14]. However, there are not enough referable data for the elderly with VAP due to antimicrobial-resistant hvKp.

To date, no data about the clinical and microbiological characteristics of VAP caused by the hvKp genotype in the elderly have been demonstrated. The aim of this study was to further investigate the clinical characteristics of elderly patients with hvKp infection and microbiological features and the epidemiology of multi-centre hvKp. Therefore, we conducted a retrospective study in two tertiary hospitals focusing on the genotype of hvKp (defined as aerobactin positive) in China.

\section{Methods}

\section{Patients}

A retrospective study was conducted on $73 \mathrm{Kp}$ culture-positive patients diagnosed from November 2008 to December 2017 at two tertiary hospitals: Beijing Tsinghua Changgung Hospital and Chinese PLA General
Hospital. These two hospitals are located in Beijing, and the capacity of the hospitals is more than 800 beds. Patients currently receiving mechanical ventilation were included in this study. The definition of VAP is according to the 2016 ATS guideline [15]. Standard bronchoalveolar lavage and aspiration were achieved. The clinical characteristics, including underlying disease, infection type, nutritional status, mortality in 30 days, and sequential organ failure assessment (SOFA) were collected. The primary outcome of this study was to investigate the 30-day mortality of hvKp infection in the elderly with VAP compared with that of the cKp group. Additionally, the white blood cell count (WBC) and neutrophil percentage (NEU\%) were applied as primary inflammatory factors. Total protein (TP) and albumin (ALB) were used to evaluate the nutrition status. The main inclusion criteria were 1) the definition of the elderly as 65 years old or older ( $\geq 65$ year) and 2) at least one $K$. pneumoniae-positive culture. The exclusion criteria was 1 ) insufficient clinical data or bacterial strain sample storage and 2) cases of co-infection.

\section{Clinical $K p$ strains}

All isolates were stored at $-80{ }^{\circ} \mathrm{C}$ and identified by the API 20 NE system and the VITEK II system. Further species identification was confirmed by $16 \mathrm{~S}$ rRNA gene sequencing. HvKp is defined as aerobactin positive. The hypermucoviscous phenotype was detected by the string test as described previously [16].

\section{Antimicrobial susceptibility testing and phenotypic detection of ESBLs}

Antimicrobial susceptibility testing was performed, and the results were interpreted according to 2017 Clinical and Laboratory Standards Institute (CLSI) guidelines. The antibiotics include amikacin, gentamicin, tobramycin, Sulbactam/sulbactam, aztreonam, cefazolin, cefepime, ceftriaxone, ceftazidime, ciprofloxacin, levofloxacin, piperacillin/tazobactam, and trimethoprim/sulfamethoxazole. ESBL was detected by an agar dilution test using ceftazidime and cefotaxime combined with clavulanate according to the CLSI guideline [10]. The definition of an MDR strain is resistance to three or more different antimicrobial categories, as described previously [17]. The carbapenem-resistant (CR) phenotype is defined as resistant to both imipenem and meropenem.

\section{Detection of virulence-associated gene}

Genomic DNA of all $K p$ isolates was extracted. $R m p A$, rmpA2, magA, aerobactin and the capsular serotype-specific (cps) genes $(K 1, K 2, K 5, K 20, K 54$, and K57) were detected by polymerase chain reaction (PCR) as described previously [10,18-20]. The primers are listed in Table 1. 
Table 1 Primers

\begin{tabular}{|c|c|}
\hline Name & sequence \\
\hline \multicolumn{2}{|l|}{ rmpA } \\
\hline Forward & 5-ACTGGGCTACCTCTGCTTCA-3 \\
\hline Reverse & 5-CTTGCATGAGCCATCTTTCA-3 \\
\hline \multicolumn{2}{|l|}{ rmpA2 } \\
\hline Forward & 5-CTTTATGTGCAATAAG-GATGTT-3 \\
\hline Reverse & 5-CCTCCTGGAGAGTAAGCATT-3 \\
\hline \multicolumn{2}{|l|}{$\operatorname{mag} A$} \\
\hline Forward & 5-GGTGCTCTITACATCATTGC-3 \\
\hline Reverse & 5-GCAATGGCCATTTGCGTTAG-3 \\
\hline \multicolumn{2}{|l|}{ aerobactin } \\
\hline Forward & 5-GCATAGGCGGATACGAACAT-3 \\
\hline Reverse & 5-CACAGGGCAATTGCTTACCT-3 \\
\hline \multicolumn{2}{|l|}{ K1 } \\
\hline Forward & 5-GTAGGTATTGCAAGCCATGC-3 \\
\hline Reverse & 5-GCCCAGGTTAATGAATCCGT-3 \\
\hline \multicolumn{2}{|l|}{ K2 } \\
\hline Forward & 5-GGAGCCATTTGAATTCGGTG-3 \\
\hline Reverse & 5-TCCCTAGCACTGGCTTAAGT-3 \\
\hline \multicolumn{2}{|l|}{ K5 } \\
\hline Forward & 5-GCCACCTCTAAGCATATAGC-3 \\
\hline Reverse & 5-CGCACCAGTAATTCCAACAG-3 \\
\hline \multicolumn{2}{|l|}{ K20 } \\
\hline Forward & 5-CCGATTCGGTCAACTAGCTT-3 \\
\hline Reverse & 5-GCACCTCTATGAACTITCAG-3 \\
\hline \multicolumn{2}{|l|}{ K54 } \\
\hline Forward & 5-CATTAGCTCAGTGGTTGGCT-3 \\
\hline Reverse & 5-GCTTGACAAACACCATAGCAG-3 \\
\hline \multicolumn{2}{|l|}{ K57 } \\
\hline Forward & 5-CGACAAATCTCTCCTGACGA-3 \\
\hline Reverse & 5-CGCGACAAACATAACACTCG-3 \\
\hline \multicolumn{2}{|l|}{ rрoB } \\
\hline Forward & 5-GGCGAAATGGCWGAGAACCA-3 \\
\hline Reverse & 5-GAGTCTTCGAAGTTGTAACC-3 \\
\hline \multicolumn{2}{|l|}{ gapA } \\
\hline Forward & 5-TGAAATATGACTCCACTCACGG-3 \\
\hline Reverse & 5-CTTCAGAAGCGGCTITGATGGCTT-3 \\
\hline \multicolumn{2}{|l|}{ mdh } \\
\hline Forward & 5-TGAAATATGACTCCACTCACGG-3 \\
\hline Reverse & 5-CTTCAGAAGCGGCTTTGATGGCTT-3 \\
\hline \multicolumn{2}{|l|}{ pgi } \\
\hline Forward & 5-GAGAAAAACCTGCCTGTACTGCTGGC-3 \\
\hline Reverse & 5-CGCGCCACGCTITATAGCGGTTAAT-3 \\
\hline \multicolumn{2}{|l|}{ phoE } \\
\hline Forward & 5-ACCTACCGCAACACCGACTTCTTCGG-3 \\
\hline
\end{tabular}

Table 1 Primers (Continued)

\begin{tabular}{ll}
\hline Name & sequence \\
\hline Reverse & 5-TGATCAGAACTGGTAGGTGAT-3 \\
infB & \\
Forward & 5-CTCGCTGCTGGACTATATTCG-3 \\
Reverse & 5-CGCTTCAGCTCAAGAACTTC-3 \\
tonB & \\
Forward & 5-CTTTATACCTCGGTACATCAGGTT-3 \\
Reverse & 5-ATTCGCCGGCTGRGCRGAGAG-3 \\
\hline
\end{tabular}

\section{Multilocus sequence typing (MLST) for Kp}

Seven housekeeping genes (gap $A, m d h$, phoE, ton $B$, infB, pgi and $r p o B$ ) were detected by PCR according to the MLST website (http://bigsdb.pasteur.fr/klebsiella/ klebsiella.html) (Table 1). Allelic profiling and sequence types (STs) were also performed using the above website. Moreover, to further distinguish the relationship among different STs, phylogenetic analysis of the seven spliced housekeeping genes for these isolates that contributed to mortality was performed by the neighbour-joining method (MEGA 6.0). The common STs, including ST1, ST11, ST37, ST258 and ST412, were used as a reference.

\section{Statistical analysis}

SPSS software (version 20.0) was employed for data analysis. Measurement data was assessed as the means \pm standard deviation (SD). The count data were analysed as percentages. Continuous variables were analysed by Student's $t$-tests and the Wilcoxon rank-sum tests. Categorical variables were analysed by the $\chi^{2}$ or Fisher's exact tests. Univariate logistic regression analysis was performed for the risk factors. A multivariable logistic regression analysis was conducted for independent risk factors (the variables with $P<0.05$ were included). All tests were 2 -tailed. A $p$-value $<0.05$ was considered significant.

\section{Results}

\section{Clinical characteristics}

Seventy-three $K p$ culture-positive patients were diagnosed at the two hospitals from November 2008 to December 2017 (Table 2). Thirty-four (46.6\%) strains were hvKp, and 37 (50.7\%) were hmvKp. Most of the patients (67, 91.8\%) presented with sepsis, and 24 patients (32.9\%) were diagnosed with septic shock. Sixty-six (90.4\%) were males, and the mean age of patients in this study was $84.96 \pm 8.33$ years. Patients with cancer $(38.2 \%$ versus $7.7 \% ; P=0.004$ ), surgery history within 1 month (23.5\% versus $5.1 \% ; P=0.023)$ and digestive diseases (29.4\% versus $10.3 \% ; P=0.046$ ) were more likely to be infected with hvKp. Although host responsibility (WBC and NEU\%) and nutritional status (TP and ALB) were 
not significantly different at the primary endpoint (30-day mortality), the SOFA scores of the patients with hvKp were notably higher at 30-day mortality (8.94 \pm 3.03 vs $6.62 \pm 2.09, P=0.000$ ) (Table 2 ).

\section{Genetic and phenotype characteristics: hvKp vs cKp}

It is noted that hypermucoviscosity was strongly clustered in the hvKp group $(P<0.001)$. Our results showed that $K 1, \operatorname{rmp} A, \operatorname{rmp} A 2$ and $m a g A$ were highly clustered in hvKp $(P<0.001)$, but $K 2, K 5, K 20, K 54$, and $K 57$ were not associated with hvKp $(P=0.073,0.213,1.000,0.096$ and 0.849 , respectively). Additionally, there is no isolate in the cKp group with $\mathrm{K} 5$ or K54 (Table 3).

\section{Antimicrobial resistance analysis and detection rate of ESBL-producing $K p$ isolates}

All Kp strains were resistant to Sulbactam. Most of the hvKp isolates represented a higher antimicrobial sensitivity rate to most of the antibiotics than cKp, except cefepime, ceftriaxone, ciprofloxacin, levofloxacin, imipenem, meropenem, and amikacin (Table 4). Although the detection rate of MDR (41.2\% vs $74.4 \%, P=0.001)$ and ESBLs

Table 2 Clinical features of patients with VAP due to hvKp

\begin{tabular}{|c|c|c|c|}
\hline Characteristic & $\operatorname{HvKp}(34)$ & cKp(39) & $P$ value \\
\hline \multicolumn{4}{|l|}{ Basic demographics } \\
\hline Age & $83.06 \pm 8.55$ & $86.62 \pm .87$ & 0.068 \\
\hline Male & $31(91.2 \%)$ & $35(89.7 \%)$ & 1.000 \\
\hline \multicolumn{4}{|l|}{ Underlying diseases } \\
\hline Pulmonary disease & $31(91.2 \%)$ & $32(82.1 \%)$ & 0.321 \\
\hline Diabetes & 19 (55.9\%) & $18(46.2 \%)$ & 0.407 \\
\hline Cardiovascular disease & $12(35.3 \%)$ & $17(43.6 \%)$ & 0.470 \\
\hline Cerebrovascular disease & $11(32.4 \%)$ & $15(38.5 \%)$ & 0.587 \\
\hline Cancer & $13(38.2 \%)$ & $3(7.7 \%)$ & 0.004 \\
\hline Surgery within 1 mo & $8(23.5 \%)$ & $2(5.1 \%)$ & 0.023 \\
\hline Digestive disease & 10 (29.4\%) & $4(10.3 \%)$ & 0.038 \\
\hline \multicolumn{4}{|l|}{ Infection type } \\
\hline Sepsis & $30(88.2 \%)$ & 37 (94.9\%) & 0.408 \\
\hline Septic shock & $16(47.1 \%)$ & $8(20.5 \%)$ & 0.016 \\
\hline \multicolumn{4}{|l|}{ Host responsibility } \\
\hline WBC & $12.57 \pm 4.47$ & $11.68 \pm 4.36$ & 0.397 \\
\hline NEU\% & $78.99 \pm 9.54$ & $77.13 \pm 7.14$ & 0.344 \\
\hline \multicolumn{4}{|l|}{ Nutrition status } \\
\hline TP & $63.38 \pm 6.34$ & $61.88 \pm 6.02$ & 0.304 \\
\hline ALB & $33.33 \pm 3.75$ & $32.51 \pm 3.39$ & 0.326 \\
\hline SOFA score & $8.94 \pm 3.03$ & $6.62 \pm 2.09$ & 0.000 \\
\hline Infection occurred in ICU & $11(32.4 \%)$ & $10(25.6 \%)$ & 0.527 \\
\hline Mortality in 30 days & $14(41.2 \%)$ & $14(35.9 \%)$ & 0.644 \\
\hline
\end{tabular}

$T P$ total protein, $A L B$ albumin, WBC white blood cell count, NEU\% neutrophils percentage
Table 3 Microbiological features of patients with VAP due to hvKp

\begin{tabular}{|c|c|c|c|}
\hline Characteristic & $\mathrm{HvKp}(34)$ & cKp(39) & $P$ value \\
\hline \multicolumn{4}{|l|}{ K serotype } \\
\hline K1 & 17 (50.0\%) & $1(2.6 \%)$ & 0.000 \\
\hline$K 2$ & 7 (2.9\%) & $2(5.1 \%)$ & 0.073 \\
\hline$K 5$ & $2(5.9 \%)$ & $0(0 \%)$ & 0.213 \\
\hline$K 20$ & $2(5.9 \%)$ & $2(5.1 \%)$ & 1.000 \\
\hline K54 & $3(8.8 \%)$ & $0(0 \%)$ & 0.096 \\
\hline K57 & $4(11.8 \%)$ & $3(7.7 \%)$ & 0.849 \\
\hline rmpA & 27 (79.4\%) & $4(10.3 \%)$ & 0.000 \\
\hline rmpA2 & $28(82.4 \%)$ & $5(12.8 \%)$ & 0.000 \\
\hline magA & 30 (88.2\%) & 17 (43.6\%) & 0.000 \\
\hline Hypermucoviscosity & $29(85.3 \%)$ & $8(20.5 \%)$ & 0.000 \\
\hline
\end{tabular}

(38.2\% vs $69.2 \%, P=0.001)$ was significantly lower than cKp, it is noted that $41.2 \%(14 / 34)$ of hvKp isolates were MDR and 38.2\% (13/34) of hvKp isolates were ESBL-producing. Moreover, 8 isolates were identified as CR-hvKp.

\section{Risk factors for VAP-hvKp}

Univariate regression analysis showed that cancer (odds ratio $[\mathrm{OR}]=7.429)$, digestive diseases $(\mathrm{OR}=3.646)$ and surgery history within 1 month $(\mathrm{OR}=5.692)$ were risk factors for hvKp infection. Multivariate analysis revealed that cancer history $(\mathrm{OR}=5.365)$ was an independent risk factor for hvKp infection (Table 5).

\section{MLST genotypic analysis}

New STs were not detected among the $73 \mathrm{Kp}$ isolates. The most prevalent ST in this study was ST23 $(n=15$; $20.5 \%)$, followed by ST412 $(n=5 ; 6.8 \%), \operatorname{ST} 17(n=4$; $5.5 \%)$, ST37 $(n=3 ; 4.1 \%)$, and ST2906 $(n=3 ; 4.1 \%)$. The above STs accounted for $41.1 \%(30 / 73)$ of the total strains. Among the prevalent STs, 73.3\% ST23 (11/15), $60.0 \%$ ST412 (3/5), and 100\% ST17 (4/4) were hvKp. The detailed features of patients with prevalent hvKp are shown in Table 6. The CR-hvKp distributed in ST17 ( $n$ $=2)$, ST23 $(n=1), \operatorname{ST} 347(n=1), \operatorname{ST} 412(n=1), \operatorname{ST} 2874$ $(n=1)$, and ST2905 $(n=1)$. The most common clone complex (CC) of the CR-hvKp was CC17 $(N=2)$. The phylogenetic tree demonstrated the relationship between the prevalent STs in our study and the common STs in the world (Fig. 1).

\section{Discussion}

To our knowledge, this is the largest multi-centre study focusing on elderly patients with VAP due to hvKp in China. Nearly half $(46.6 \%, 34 / 73)$ of the hvKp-induced VAP in the elderly patients occurred among mechanically ventilated patients. The hvKp group showed a higher 
Table 4 Antibiotics resistance: hvKp vs cKp

\begin{tabular}{llll}
\hline Antibiotic agent & HvKp(34) & cKp(39) & $P$ value \\
\hline MDR & $\mathbf{1 4}(\mathbf{4 1 . 2 \% )}$ & $\mathbf{2 9}(\mathbf{7 4 . 4 \% )}$ & $\mathbf{0 . 0 0 4}$ \\
ESBLs & $\mathbf{1 3 ( 3 8 . 2 \% )}$ & $\mathbf{2 7}(\mathbf{6 9 . 2} \%)$ & $\mathbf{0 . 0 0 8}$ \\
Ampicillin & $34(100 \%)$ & $39(100 \%)$ & $\mathrm{NA}$ \\
Amikacin & $6(17.6 \%)$ & $12(30.8 \%)$ & 0.194 \\
Gentamicin & $\mathbf{8 ( 2 3 . 5 \% )}$ & $\mathbf{2 1 ( 5 3 . 8 \% )}$ & $\mathbf{0 . 0 0 8}$ \\
Ampicillin/Sulbactam & $\mathbf{1 3 ( 3 8 . 2 \% )}$ & $\mathbf{2 7}(\mathbf{6 9 . 2} \%)$ & $\mathbf{0 . 0 0 8}$ \\
Aztreonam & $\mathbf{1 0 ( 2 9 . 4 \% )}$ & $\mathbf{2 1 ( 5 3 . 8 \% )}$ & $\mathbf{0 . 0 3 5}$ \\
Cefazolin & $\mathbf{1 4}(\mathbf{4 1 . 2} \%)$ & $\mathbf{2 8 ( 7 1 . 8 \% )}$ & $\mathbf{0 . 0 0 8}$ \\
Cefotetan & $\mathbf{8 ( 2 3 . 5 \% )}$ & $\mathbf{1 6}(\mathbf{4 1 . 0 \% )}$ & $\mathbf{0 . 1 1 2}$ \\
Cefepime & $9(26.5 \%)$ & $18(46.2 \%)$ & 0.082 \\
Ceftriaxone & $13(38.2 \%)$ & $23(59.0 \%)$ & 0.077 \\
Ceftazidime & $\mathbf{1 0 ( 2 9 . 4 \% )}$ & $\mathbf{2 3 ( 5 9 . 0 \% )}$ & $\mathbf{0 . 0 1 1}$ \\
Ciprofloxacin & $10(29.4 \%)$ & $20(51.3 \%)$ & 0.058 \\
Levofloxacin & $9(26.5 \%)$ & $19(48.7 \%)$ & 0.051 \\
Trimethoprim/Sulfamethoxazole & $\mathbf{7 ( \mathbf { 2 0 . 6 } \% )}$ & $\mathbf{2 2 ( 5 6 . 4 \% )}$ & $\mathbf{0 . 0 0 2}$ \\
Piperacillin/Tazobactam & $\mathbf{8 ( 2 3 . 5 \% )}$ & $\mathbf{1 8 ( 4 6 . 2 \% )}$ & $\mathbf{0 . 0 4 4}$ \\
Imipenem & $8(23.5 \%)$ & $10(25.6 \%)$ & 0.835 \\
Meropenem & $9(26.5 \%)$ & $10(25.6 \%)$ & 0.936 \\
Tobramycin & $\mathbf{1 0 ( 2 9 . 4 \% )}$ & $\mathbf{2 1 ( 5 3 . 8 \% )}$ & $\mathbf{0 . 0 2 7}$ \\
\hline
\end{tabular}

severity of disease than did the cKp group. The primary virulence factors, such as $\mathrm{K} 1, \mathrm{rmpA} / \mathrm{A} 2$, and magA, were highly clustered in the hvKp group. ST23 was the most prevalent ST in two hospitals. However, the ST23 isolated in different hospitals was not highly associated with the aerobactin genetic background or hypermucoviscous phenotype, and it was concluded that just relying on STs to identify hvKp may be not appropriate. In our study, $50.7 \%$ of $K$. pneumonia isolates were identified as hypermucoviscous by the string test experiment, which is higher than that in a previous retrospective study conducted in a single centre in China, with a prevalence of $33 \%$ [16]. Thus, plasmid type, biofilm production, serotypes and the ability to induce inflammatory factors may be needed to further define hvKp. The prevalence of hvKp may be incorrectly estimated because of the lack of definite and objective diagnostic methods.

Aerobactin was considered a potential virulence trait for the hvKp genotype in in vitro and in vivo models [3]. Importantly, previous studies showed that the hvKp genotype (aerobactin-positive) often induced more serious invasive infection $[10,11,21]$. Previous studies reported that the detection rate of hvKp ranged from 4.5 to $37.8 \%$ in various clinical specimens $[7,10,16,19,22]$. In this study, 34 isolates $(46.6 \%)$ were defined as hvKp due to aerobactin-positivity, which is the highest incidence for hospital-acquired infection (HAI) caused by the hvKp genotype. It is noted that the detection of the hvKp genotype in this study was also higher than that in previous multicentre studies(37.8\%) based on adults [10] and a single centre retrospective study (28.6\%) focusing on VAP [23]. It is concluded that hvKp may emerge as a major pathotype for elderly with VAP in the two hospitals.

Additionally, capsular serotype-specific genes are essential factors for $K$. pneumoniae. To date, various types of K-antigen have been reported [20, 24, 25]. In Asia, K1 and $\mathrm{K} 2$ are the most important elements, inducing severe infections. However, they are not the unique trait for hvKp [26-29]. Previous studies demonstrated that K1 distribution in hvKp ranged from 23 to $98 \%$ in various specimens in various populations [29-33]. The detection rate of $\mathrm{K} 2$ in hvKp ranged from 10 to $46 \%$ [16, $31,33]$. Moreover, a previous single centre study focusing on VAP showed that $K 1$ and $K 2$ among hvKp accounted for 42.9 and $21.4 \%$, respectively [23]. The above data implied that $\mathrm{K} 1 \mathrm{might}$ be highly associated with hvKp compared with K2. In our study, half of the hvKp (50\%) isolates were associated with $K 1$, and $K 2$ was detected in $7 \mathrm{hvKp}$ isolates $(2.9 \%)$, which was consistent with previous studies. In addition to $K 1$ and $K 2$, there were no significant difference between hvKp and cKp in $K 5, K 20, K 54$, and $K 57$ due to small specimens. Moreover, $r m p A / r m p A 2$ and $m a g A$ genes responsible for

Table 5 Risk factor for hvKp vs cKp

\begin{tabular}{|c|c|c|c|c|}
\hline Variable & Univariate OR $(95 \% \mathrm{Cl})$ & $P$ value & Multivariate OR $(95 \% \mathrm{Cl})$ & $P$ value \\
\hline Infection occurred in ICU & $1.387(0.502-3.832)$ & 0.528 & & \\
\hline Male & $1.181(0.245-5.694)$ & 0.836 & & \\
\hline Pulmonary diseases & $2.260(0.536-9.539)$ & 0.267 & & \\
\hline Diabetes & $1.478(0.586-3.725)$ & 0.408 & & \\
\hline Cardiovascular disease & $0.706(0.274-1.818)$ & 0.471 & & \\
\hline Cerebrovascular disease & $0.765(0.291-2.010)$ & 0.587 & & \\
\hline Cancer & $7.429(1.895-29.114)$ & 0.004 & $5.365(1.199-24.007)$ & 0.028 \\
\hline Surgery within $1 \mathrm{mo}$ & $5.692(1.117-29.013)$ & 0.036 & & \\
\hline Digestive diseases & $3.646(1.023-12.990)$ & 0.046 & $3.713(0.970-14.212)$ & 0.055 \\
\hline
\end{tabular}


Table 6 Features of patients with prevalent $\operatorname{hvKp}(n \geq 3)$

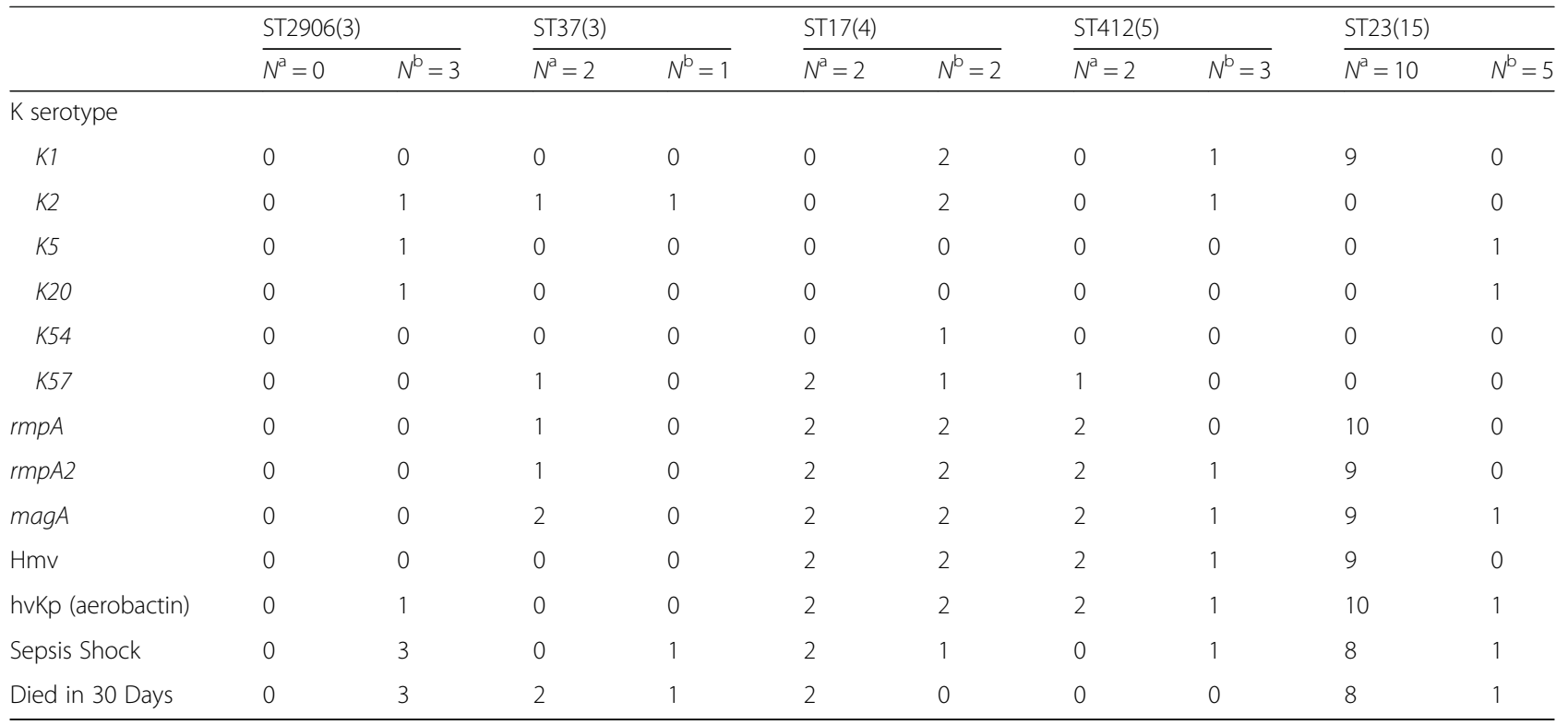

$H m v$ hypermucoviscosity, $a$ and $b$ different hospital

the hypermucoviscous phenotype were proposed as virulence factors in addition to major cps $K 1 / K 2$ [5, 19, 30, 34]. In our study, 2 hvKp isolates inserted rmpA did not show hypermucoviscosity. It is implied that there might exist other regulatory mechanisms for hypermucoviscosity. Importantly, our results are consistent with previous study: most of the dominant virulence-associated factors (K1, rmpA/A2 and magA) and hypermucoviscosity are highly clustered in the hvKp group [10].

Although there was no significant difference in sepsis incidence and mortality between the two VAP groups, the incidence of sepsis shock and SOFA score was higher than those in the cKp group. Thus, to further understand the risk factors for hvKp, timely and appropriately prevention is essential. Our results showed that

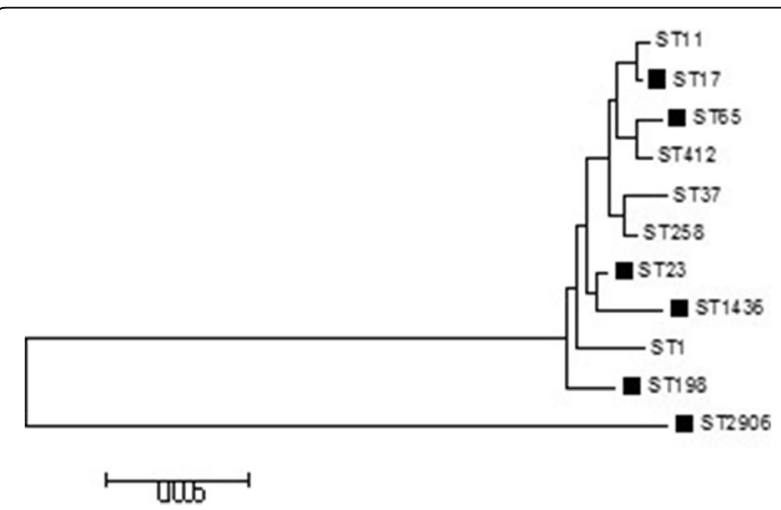

Fig. 1 Neighbour-joining dendrogram of concatenated sequences of seven housekeeping genes from the MLST database for prevalent STs in our study and the common STs in the world. (Black solid box represents death in our study) elderly patients with cancer, digestive disease and surgery history within 1 month were more likely to be infected with hvKp. Moreover, cancer history was an independent risk factor for VAP-hvKp infection in the elderly, and cancer patients should be monitored more intensively to prevent infection due to immunocompromise, which may also be a critical factor for VAP-hvKp [10]. Previous multi-centre study of the hvKp genotype based on adults in China concluded that diabetes and cancer were independent risk factors for hvKp [10]. However, the incidence of diabetes $(50.7 \%, 37 / 73)$ in our study is far more than previous multi-centre study focused on adults $(17.0 \%, 39 / 230)$ [10]. This may be the characteristics of the elderly who often suffer from various underlying diseases. Additionally, a previous study concluded that major histocompatibility complex (MHC) variants, nutritional status, and the gut microbiota might be potential host factors for improving the understanding of the hypervirulence phenomenon [9]. Although the incidence of septic shock and SOFA score in our study were higher than those of the cKp group, there was no significant difference in the nutritional status (TP and ALB) and inflammatory reaction (WBC and NEU\%), which was consistent with a previous study [23]. Thus, other inflammatory factors may be needed to screen as a marker for elderly patients with VAP-hvKp.

Previous studies revealed that most $\mathrm{cKp}$ and antimicrobial-resistant patterns were overlapping, which is not frequent in hvKp $[10,16]$. In this study, most of the hvKp isolates were sensitive to most of the above antibiotics. However, in the hvKp group, the number of ESBL-hvKp (41.2\%) and MDR-hvKp (38.2\%) is 
significantly higher than that in previous studies [10,23]. It is alarming that MDR-hvKp may prefer to induce VAP in the elderly. Moreover, eight CR-hvKp isolates were identified as another "superbug". Taken together, these data revealed that MDR-hvKp is emerging among elderly patients with VAP, which needs to be confirmed by further investigations in larger populations. The CR-hvKp isolates were not detected in the nosocomial environment by routine nosocomial infection surveillance. Additionally, the composition of the gut microbiota was unclear because anal swabs were not applied for nosocomial infection surveillance. Previous study suggested that wards previously infected with CR-hvKp should be disinfected and left unoccupied for more than 2 weeks [12]. It may be a good choice to prevent fatal outbreaks of infection, especially in critically ill and immunocompromised elderly patients with mechanical ventilation.

The main limitation in our study is that it is a retrospective study from over 10 years. Although this study included two large tertiary hospitals, fewer patients with VAP-hvKp were included. However, this study is the largest cohort to investigate the clinical and microbiological characteristics of elderly patients with VAP-hvKp to now. Additionally, most of the key inflammatory factors, nutrition statue markers and environmental samples were not achieved. Moreover, the expression of aerobactin is unclear. Whole genome sequencing, transcriptomics and proteomics may be needed for further study to identify genetic expression.

\section{Conclusions}

HvKp is emerging as a common pathogen of VAP in the elderly in China. There is various epidemiology of VAP-hvKp in different hospitals. Although STs may not be associated with hvKp, ST23, ST37 and ST2906 are more likely to induce poor prognosis. Although the definition of hvKp is still controversial, the hvKp genotype is more likely to cause septic shock and a higher SOFA score. The emerging MDR-hvKp, especially CR-hvKp, will be a "superbug", which is a great challenge for clinicians. It is essential to enhance clinical awareness and infection surveillance for various hvKp infections, especially in immunocompromised elderly patients.

\section{Acknowledgements}

We thank the team of curators at the Institut Pasteur MLST and whole genome MLST databases for curating the data and making them publicly available at http://bigsdb.pasteur.fr

\section{Funding}

This work was supported by the China postdoctoral science foundation (grant number 2014 M562610); the Excellent Young Program of the Organization Department of Beijing Municipal Party Committee (grant number 2016000057592G258).

\section{Availability of data and materials}

The datasets used and/or analysed during the current study are available from the corresponding author on reasonable request.

\section{Authors' contributions}

$J G$ and $C L$ were responsible for study design and article writing. $C L$ and JG collected clinical data and performed PCR and statistical analyses. JG performed critical data review. All authors read and approved the final version of the manuscript.

\section{Ethics approval and consent to participate}

Informed consent was not needed due to the retrospective nature of the study. The study was approved by the Chinese PLA General Hospital Ethics Committee, and the Guidelines for Human Experimentation (PR. China) were followed throughout.

\section{Consent for publication}

Not applicable.

\section{Competing interests}

The authors declare that they have no competing interests.

\section{Publisher's Note}

Springer Nature remains neutral with regard to jurisdictional claims in published maps and institutional affiliations.

\section{Author details}

'Department of Respiratory Medicine, Peking Union Medical College, Chinese Academy of Medical Sciences, China-Japan Friendship Hospital, Beijing, China. ${ }^{2}$ Department of Respiratory Medicine, School of Clinical Medicine, Beijing Tsinghua Changgung Hospital, Tsinghua University, Beijing, China. ${ }^{3}$ Department of Geriatric Respiratory Medicine, Chinese PLA General Hospital, Beijing, China.

Received: 14 March 2018 Accepted: 26 June 2018

Published online: 03 August 2018

\section{References}

1. Timsit JF, Esaied W, Neuville M, Bouadma L, Mourvllier B. Update on ventilator-associated pneumonia. F1000Res. 2017;6:2061.

2. Torres A, Niederman MS, Chastre J, Ewig S, Fernandez-Vandellos P, Hanberger H, Kollef M, Li Bassi G, Luna CM, Martin-Loeches I, et al. International ERS/ESICM/ESCMID/ALAT guidelines for the management of hospital-acquired pneumonia and ventilator-associated pneumonia: guidelines for the management of hospital-acquired pneumonia (HAP)/ ventilator-associated pneumonia (VAP) of the European Respiratory Society (ERS), European Society of Intensive Care Medicine (ESICM), European Society of Clinical Microbiology and Infectious Diseases (ESCMID) and Asociacion Latinoamericana del Torax (ALAT). Eur Respir J. 2017;50:1700582.

3. Russo TA, Olson R, MacDonald U, Beanan J, Davidson BA. Aerobactin, but not yersiniabactin, salmochelin, or enterobactin, enables the growth/ survival of hypervirulent (hypermucoviscous) Klebsiella pneumoniae ex vivo and in vivo. Infect Immun. 2015:83(8):3325-33.

4. Shon AS, Bajwa RP, Russo TA. Hypervirulent (hypermucoviscous) Klebsiella pneumoniae: a new and dangerous breed. Virulence. 2013; 4(2):107-18

5. Siu LK, Yeh KM, Lin JC, Fung CP, Chang FY. Klebsiella pneumoniae liver abscess: a new invasive syndrome. Lancet Infect Dis. 2012;12(11):881-7.

6. Pomakova DK, Hsiao CB, Beanan JM, Olson R, MacDonald U, Keynan Y, Russo TA. Clinical and phenotypic differences between classic and hypervirulent Klebsiella pneumonia: an emerging and under-recognized pathogenic variant. Eur J Clin Microbiol Infect Dis. 2012;31(6):981-9.

7. Zhang Y, Zeng J, Liu W, Zhao F, Hu Z, Zhao C, Wang Q, Wang X, Chen $\mathrm{H}$, Li H, et al. Emergence of a hypervirulent carbapenem-resistant Klebsiella pneumoniae isolate from clinical infections in China. J Infect. 2015;71(5):553-60.

8. Lin YC, Lu MC, Tang HL, Liu HC, Chen CH, Liu KS, Lin C, Chiou CS, Chiang MK, Chen CM, et al. Assessment of hypermucoviscosity as a virulence factor for experimental Klebsiella pneumoniae infections: comparative 
virulence analysis with hypermucoviscosity-negative strain. BMC Microbiol. 2011;11:50.

9. Catalan-Najera JC, Garza-Ramos U, Barrios-Camacho H. Hypervirulence and hypermucoviscosity: two different but complementary Klebsiella spp. phenotypes? Virulence. 2017:1-13.

10. Zhang Y, Zhao C, Wang Q, Wang X, Chen H, Li H, Zhang F, Li S, Wang $\mathrm{R}$, Wang $\mathrm{H}$. High prevalence of Hypervirulent Klebsiella pneumoniae infection in China: geographic distribution, clinical characteristics, and antimicrobial resistance. Antimicrob Agents Chemother. 2016;60(10): 6115-20.

11. Russo TA, Olson R, Macdonald U, Metzger D, Maltese LM, Drake EJ, Gulick AM. Aerobactin mediates virulence and accounts for increased siderophore production under iron-limiting conditions by hypervirulent (hypermucoviscous) Klebsiella pneumoniae. Infect Immun. 2014;82(6): 2356-67.

12. Gu D, Dong N, Zheng Z, Lin D, Huang M, Wang L, Chan EW, Shu L, Yu J, Zhang R, et al. A fatal outbreak of ST11 carbapenem-resistant hypervirulent Klebsiella pneumoniae in a Chinese hospital: a molecular epidemiological study. Lancet Infect Dis. 2018;18(1):37-46

13. Gu DX, Huang YL, Ma JH, Zhou HW, Fang Y, Cai JC, Hu YY, Zhang R. Detection of Colistin resistance gene mcr-1 in Hypervirulent Klebsiella pneumoniae and Escherichia coli isolates from an infant with diarrhea in China. Antimicrob Agents Chemother. 2016;60(8):5099-100.

14. Zhang R, Lin D, Chan EW, Gu D, Chen GX, Chen S. Emergence of Carbapenem-resistant serotype K1 Hypervirulent Klebsiella pneumoniae strains in China. Antimicrob Agents Chemother. 2015;60(1):709-11.

15. Kalil AC, Metersky ML, Klompas M, Muscedere J, Sweeney DA, Palmer LB, Napolitano LM, O'Grady NP, Bartlett JG, Carratala J, et al. Management of Adults with Hospital-acquired and Ventilator-associated Pneumonia: 2016 clinical practice guidelines by the Infectious Diseases Society of America and the American Thoracic Society. Clin Infect Dis. 2016;63(5):e61-e111.

16. Li W, Sun G, Yu Y, Li N, Chen M, Jin R, Jiao Y, Wu H. Increasing occurrence of antimicrobial-resistant hypervirulent (hypermucoviscous) Klebsiella pneumoniae isolates in China. Clin Infect Dis. 2014;58(2):225-32.

17. Magiorakos AP, Srinivasan A, Carey RB, Carmeli Y, Falagas ME, Giske CG, Harbarth S, Hindler JF, Kahlmeter G, Olsson-Liljequist B, et al. Multidrugresistant, extensively drug-resistant and pandrug-resistant bacteria: an international expert proposal for interim standard definitions for acquired resistance. Clin Microbiol Infec. 2012;18(3):268-81.

18. Choi MJ, Ko KS. Loss of hypermucoviscosity and increased fitness cost in colistin-resistant Klebsiella pneumoniae sequence type 23 strains. Antimicrob Agents Chemother. 2015;59(11):6763-73.

19. Yu WL, Ko WC, Cheng KC, Lee HC, Ke DS, Lee CC, Fung CP, Chuang YC. Association between rmpA and magA genes and clinical syndromes caused by Klebsiella pneumoniae in Taiwan. Clin Infect Dis. 2006;42(10):1351-8.

20. Cheng NC, Yu YC, Tai HC, Hsueh PR, Chang SC, Lai SY, Yi WC, Fang CT. Recent trend of necrotizing fasciitis in Taiwan: focus on monomicrobial Klebsiella pneumoniae necrotizing fasciitis. Clin Infect Dis. 2012;55(7):930-9.

21. Nassif $X$, Sansonetti PJ. Correlation of the virulence of Klebsiella pneumoniae $\mathrm{K} 1$ and $\mathrm{K} 2$ with the presence of a plasmid encoding aerobactin. Infect Immun. 1986;54(3):603-8.

22. Lee HC, Chuang YC, Yu WL, Lee NY, Chang CM, Ko NY, Wang LR, Ko WC. Clinical implications of hypermucoviscosity phenotype in Klebsiella pneumoniae isolates: association with invasive syndrome in patients with community-acquired bacteraemia. J Intern Med. 2006;259(6):606-14.

23. Yan Q, Zhou M, Zou M, Liu WE. Hypervirulent Klebsiella pneumoniae induced ventilator-associated pneumonia in mechanically ventilated patients in China. Eur J Clin Microbiol Infect Dis. 2016;35(3):387-96.

24. Pan YJ, Fang HC, Yang HC, Lin TL, Hsieh PF, Tsai FC, Keynan Y, Wang JT. Capsular polysaccharide synthesis regions in Klebsiella pneumoniae serotype K57 and a new capsular serotype. J Clin Microbiol. 2008:46(7): 2231-40.

25. Chuang YP, Fang CT, Lai SY, Chang SC, Wang JT. Genetic determinants of capsular serotype K1 of Klebsiella pneumoniae causing primary pyogenic liver abscess. J Infect Dis. 2006;193(5):645-54.

26. Yeh KM, Kurup A, Siu LK, Koh YL, Fung CP, Lin JC, Chen TL, Chang FY, Koh TH. Capsular serotype K1 or K2, rather than magA and rmpA, is a major virulence determinant for Klebsiella pneumoniae liver abscess in Singapore and Taiwan. J Clin Microbiol. 2007;45(2):466-71.

27. Brisse $S$, Fevre C, Passet $V$, Issenhuth-Jeanjean S, Tournebize R, Diancourt L, Grimont P. Virulent clones of Klebsiella pneumoniae: identification and evolutionary scenario based on genomic and phenotypic characterization. PLoS One. 2009;4(3):e4982.

28. Fang CT, Lai SY, Yi WC, Hsueh PR, Liu KL, Chang SC. Klebsiella pneumoniae genotype K1: an emerging pathogen that causes septic ocular or central nervous system complications from pyogenic liver abscess. Clin Infect Dis. 2007:45(3):284-93.

29. Lin JC, Koh TH, Lee N, Fung CP, Chang FY, Tsai YK, Ip M, Siu LK. Genotypes and virulence in serotype K2 Klebsiella pneumoniae from liver abscess and non-infectious carriers in Hong Kong, Singapore and Taiwan. Gut Pathogens. 2014;6:21.

30. Fang $C T$, Chuang YP, Shun $C T$, Chang SC, Wang JT. A novel virulence gene in Klebsiella pneumoniae strains causing primary liver abscess and septic metastatic complications. J Exp Med. 2004;199(5):697-705.

31. Liu YM, Li BB, Zhang YY, Zhang W, Shen H, Li H, Cao B. Clinical and molecular characteristics of emerging hypervirulent Klebsiella pneumoniae bloodstream infections in mainland China. Antimicrob Agents Chemother. 2014:58(9):5379-85.

32. Compain F, Babosan A, Brisse S, Genel N, Audo J, Ailloud F, Kassis-Chikhan $\mathrm{N}$, Arlet $\mathrm{G}$, Decre D. Multiplex PCR for detection of seven virulence factors and K1/K2 capsular serotypes of Klebsiella pneumoniae. J Clin Microbiol. 2014:52(12):4377-80.

33. Lee SS, Chen YS, Tsai HC, Wann SR, Lin HH, Huang CK, Liu YC. Predictors of septic metastatic infection and mortality among patients with Klebsiella pneumoniae liver abscess. Clin Infect Dis. 2008;47(5):642-50.

34. Struve C, Bojer M, Nielsen EM, Hansen DS, Krogfelt KA. Investigation of the putative virulence gene magA in a worldwide collection of 495 Klebsiella isolates: magA is restricted to the gene cluster of Klebsiella pneumoniae capsule serotype K1. J Med Microbiol. 2005;54(Pt 11):1111-3.

\section{Ready to submit your research? Choose BMC and benefit from:}

- fast, convenient online submission

- thorough peer review by experienced researchers in your field

- rapid publication on acceptance

- support for research data, including large and complex data types

- gold Open Access which fosters wider collaboration and increased citations

- maximum visibility for your research: over $100 \mathrm{M}$ website views per year

At BMC, research is always in progress.

Learn more biomedcentral.com/submissions 